\title{
A note on the penalty correction method
}

\author{
W. HACKBUSCH
}

Die Methode der Penalty-Defektkorrektur von J. PAScrak führt ein inhomogenes Dirichletsches Randwertproblem auf eine Folge von Problemen finiter Elemente mit natürlicher: Randwertbedingungen zurück. In der Arbeit wird gezeigt, daß die Methode der PenaltyDefektkorrektur ein Spezialfall der iterierten Defektkorrektur ist. Dabei gelingen Fehlerabschätzungen bereits unter sehr schwachen Voraussetzungen.

Метод штрафных поправок Ј. РАзсыл сводит решение неоднородной краевой задачи Дирихле к последовательности задач конечных әлементов с естественными краевыми условнями. В работе показывается, что метод штрафных поправок есть частныи случай итерированной поправки дефекта. При этом оценки погрешности получены при более слабых предположениях.

The penalty correction method of J. Pascrar reduces the solution of an inhomogeneous Dirichlet boundary value problem to a sequence of finite element problems with natural boundary conditions. We show that the penalty correction method is a special case of the iterated defect correction. Error estimates are proved under weaker assumptions.

\section{Introdnction}

We consider an elliptic boundary value problem with an inhomogeneous Dirichlet condition. While inhomogeneous natural boundary conditions can easily be treated by finite element discretizations, the given problem is more difficult. Recently, J. Pasciak [6] proposed a penalty correction method (PCM) that solves the Dirichlet problem by a small sequence of equations with natural boundary conditions. Frror estimates can be proved under very weak assumptions.

The first purpose of this paper is to show that the penalty correction method is a special case of the more general iterated defect correction (IDC) as described by the author (cf. [2]). The error estimates of the PCM follow from those of the IDC.

Secondly we weaken the assumptions. PAsclak required the differential operator to be symmetric and uniformly positive definite. Here we prove the same results without these assumptions.

In Section 2 the iterated defect correction (IDC) is described. Section 3 contains the penalty correction method (PCM) and the corresponding error estimates. The proof in Section 3.3 shows that PCM can be reformulated as IDC. Section 3.3 contains a device for the numerical solving of the discrete equations by the multi-grid iteration. The last section contains proofs of the foregoing lemmata.

\section{Iterated defect correction method}

In this section we repeat the iterated defect correction method for a general (abstract) equation. The iterated defect correction method is based on two discretizations. The basic discretization of accuracy $\mathcal{O}\left(h^{x}\right)$ is solved several times with different right- 
hand sides. By corrections with respect to the second discretization of a higher accuracy $\mathcal{O}\left(h^{x^{*}}\right)$, the iteration yields a solution of the same order $\mathcal{O}\left(h^{x^{*}}\right)$.

Consider the abstract linea $\mathrm{r}^{1}$ ) equation

$$
F x=y \text {. }
$$

Assume that there are two different discretizations

$$
\begin{aligned}
& F_{h} x_{h}=y_{h}:=R_{h}{ }^{Y} y, \\
& F_{h}{ }^{\prime} x_{h}{ }^{\prime}=y_{h}{ }^{\prime}:=R_{h}{ }^{\prime} y,
\end{aligned}
$$

corresponding to the discretization parameter $h \in H$, where $O \in \bar{H}$. The first discretization (2.2) should easily to be solved, while the second one has a higher order of consistency. The second discretization may be instable, even unsolvable.

The iterated defect correction is advantageous if the numerical solution of $F_{h}^{\prime} x_{h}{ }^{\prime}=y_{h}^{\prime}$ is much more difficult than the solution of $F_{h} x_{h}=y_{h}$, or if $F_{h}^{\prime}$ is instable or not invertible. There are many situations, in particular in the field of partial differential equations, where discretizations of higher order of consistency lead to very difficult equations or even to instable problems.

The iterated defect correction ${ }^{2}$ ) (IDC) is defined by

$$
x_{h}^{1}:=F_{h}^{-1} y_{h}, \quad x_{h}^{i+1}:=x_{h}{ }^{i}-F_{h}{ }^{-1}\left(F_{h}{ }^{\prime} x_{h}{ }^{i}-y_{h}{ }^{\prime}\right) \text {. }
$$

In general the sequence $\left\{x_{h}{ }^{i}\right\}$ does not converge, but the order of consistency increases with $i$ till the order of $(2.3)$ is reached.

The characteristic feature of the analysis of the IDC is the use of norms corresponding to varying orders of differentiability. During the iteration the order of differentiability decreases, while the exponent of the discretization parameter $l$ appearing in the right-hand side of the error estimate increases.

Let $X^{a}$ ( $\alpha$ varying) be a scale of Banach spaces containing the solution $u$ of (2.1). Usually, $\alpha$ is related to the order of differentiability. Examples are the Hölder spaces $X^{a}=C^{a}(\Omega)$ or the Sobolev spaces $X^{a}=H^{\alpha}(\Omega)$. Similarly, the right-hand side of (2.1) is contained in $Y^{\alpha}$. The discrete functions $u_{h}$ and $f_{h}$ belong the some vector spaces. Endowing these vector spaces with analogous norms we obtain the scales of Banach spaces $X_{h}{ }^{a}$ and $Y_{h}{ }^{\alpha}$. For details compare [2]. We assume $\|\cdot\|_{x_{h}} \leqq C(s, t)\|\cdot\|_{X_{h^{a}}}$ for $s \leqq t$.

The following stability and consistency assumptions are needed in Theorem 2.1. The stability condition reads as

$$
\left\|F_{h}^{-1} y_{h}\right\|_{X_{h}{ }^{a-\sigma}} \leqq C\left\|y_{h}\right\|_{Y_{h}{ }^{a}} \text { for all } y_{h} \in Y_{h}{ }^{a}, h \in H \text {, and some } \sigma \geqq 0 \text {. }
$$

$C$ always denotes a generic constant independent of $h$. Assume that (2.2) and (2.3) are consistent of the orders $x$ and $x^{\prime}>x$, respectively:

$$
\text { - }\left\|\left(R_{h}{ }^{Y} F-F_{h} R_{h}{ }^{X}\right) x\right\|_{Y_{h}^{\alpha}} \leqq C h^{\beta}\|x\|_{X^{\alpha+\beta}}
$$

for $\beta \in[0, x]$ and all $x \in X^{\alpha+\beta}, h \in H$,

$$
\left\|\left(R_{h}{ }^{\prime} F-F_{h}{ }^{\prime} R_{h}{ }^{X}\right) x\right\|_{Y_{h}{ }^{a}} \leqq C h^{\beta}\|x\|_{X^{\alpha} \div \beta}
$$

1) It is not difficult to extend the defect correction method to non-linear problems (cf. STETTER [7]).

2) More references to IDC are given in [2] and [7]. 
for $\beta \in\left[0, x^{\prime}\right]$ and all $x \in X^{\alpha+\beta}, h \in H . R_{h}{ }^{x}: X^{s} \rightarrow X_{h}^{8}$ denotes a restriction to $X_{h}{ }^{8}$. $R_{h}{ }^{Y}$ and $R_{h}{ }^{\prime Y}$ are restrictions from $Y^{a}$ to $Y_{h}{ }^{8}$. An additional condition is

$$
\left\|\left(F_{h}^{\prime}-F_{h}\right) x_{h}\right\|_{Y_{A}^{a}} \leqq C h^{\beta}\left\|x_{h}\right\|_{X_{h}^{\alpha+\beta}}
$$

for $\beta \in[0, \varkappa]$ and for all $x_{h} \in X_{h}{ }^{\alpha+\beta}, h \in H$. The following theorem is proved in [2].

Theorem 2.1: Set $t=s+i(\sigma+\varkappa)$. Assume (2.5) for all $\alpha \in[s+\sigma, t-\chi]$, (2.6a) for $\alpha=t-x$ and $\beta=x,(2.6 \mathrm{~b})$ for all $\alpha \in\left[\max \left(s+\sigma, t-x^{\prime}\right), t-x\right]$ and $\alpha+\beta=t,(2.7)$ for all $\alpha \in[s+\sigma, t-\psi-\sigma]$ and $\beta=x$. Then the $i$-th iterate $x_{h}{ }^{i}$ of the defect correction (2.4) satisfies

$$
\left\|x_{h}^{i}-x_{h}^{*}\right\|_{x_{n^{i}}} \leqq C(i) h^{\min \left(x^{i}, i x\right)}\left\|x^{*}\right\|_{X^{i}}
$$

where $x_{h}{ }^{*}=R_{h} x^{*}$ and $x^{*}$ solution of (2.1).

If $s, \sigma, x$, and $x^{\prime}$ are integers, the values of $\alpha, \beta$ in (2.5)-(2.7) can be restricted to integers in the respective intervals.

Theorem 2.1 will be applied to the special problem described in the next section. In Section 3.4 the definitions of the spaces $X^{\alpha}$ etc. and of the mappings $F, F_{h}, F_{h}{ }^{\prime}$ are given.

\section{Penalty correction method}

\subsection{Description}

Consider the elliptic problem

$$
\begin{aligned}
& L u=f \quad \text { in } \Omega, \\
& u=g \quad \text { on } \Gamma=\partial \Omega,
\end{aligned}
$$

where without loss of generality $L$ is a differential operator of second orde. Assume that Green's formula

$$
\left.a(u, v)=(L u, v)+\langle B u, v\rangle=\left(u, L^{*} v\right)+\langle u, C v\rangle \quad\left(u, v \in C^{\infty} \Omega\right)\right)
$$

nolds for a bilinear form $a(\cdot, \cdot) \cdot(\cdot, \cdot)$ and $\langle\cdot, \cdot\rangle$ are the scalar products in $L^{2}(\Omega)$ and $L^{2}(\Gamma)$, respectively. $B$ and $C$ denote boundary operators of first order. For $h \in H:=\left(0, h_{0}\right]$ define

$$
A_{h}(u, v):=a(u, v)+h^{-1}\langle u, v\rangle \text {. }
$$

Then the variational problem

$$
A_{h}(u, v)=(f, v)+h^{-1}\langle g, v\rangle \quad \text { for all } v \in H^{1}(\Omega)
$$

corresponds to the differential equation (3.1a) with the boundary condition

$$
u+h B u=g \text { on } \Gamma \text {. }
$$

Assume that $S_{h}$ is an approximating subspace of $H^{1}(\Omega)$ of order $m$ :

$$
\inf _{\phi_{h} \in S_{h}}\left\|u-\varphi_{h}\right\|_{1} \leqq C h^{s-1}\|u\|_{s} \quad \text { for all } u \in H^{s}(\Omega), s \in[1, m] .
$$

The norms are defined below. For simplicity we assume that $m$ is an integer $\geqq 2$. 
Algorithm (cf. PAScLAK [6]): The penaity correction method starts with the discrete solution of (3.3) and continues with correction of the boundary condition. The iterates $u_{h}^{i} \in S_{h}$ are defined by

$$
\begin{aligned}
& A_{h}\left(u_{h}^{1}, v_{h}\right)=\left(f, v_{h}\right)+h^{-1}\left\langle g, v_{h}\right\rangle \quad \text { for all } v_{h} \in S_{h}, \\
& A_{h}\left(u_{h}^{i+1}-u_{h}^{i}, v_{h}\right)=h^{-1}\left\langle g-u_{h}^{i}, v_{h}\right\rangle \quad \text { for all } v_{h} \in S_{h}, i=1,2, \ldots
\end{aligned}
$$

For the numerical solution of $(3.5 a, b)$ compare Section 3.3.

\subsection{Error estimates}

Let $H^{s}(\Omega)$ and $H^{s}(\Gamma)(s \geqq 0)$ be the Sobolev spaces of order 8 with norms denoted by $\|\cdot\|_{s}$ and $|\cdot|_{s}$, respectively. $H^{-8}(\Omega)$ and $H^{-8}(\Gamma)(s \geqq 0)$ are defined as the respective dual spaces with the dual norms $\|\cdot\|_{-s},|\cdot|_{-s}$.

The bilinear form $a(\cdot, \cdot)$ is assumed to be continuous and $H^{1}(\Omega)$-coercive:

$$
\begin{aligned}
& |a(u, v)| \leqq C\|u\|_{1}\|v\|_{1} \quad \text { for all } u, v \in H^{1}(\Omega), \\
& a(u, u) \geqq \varepsilon\|u\|_{1}^{2}-C\|u\|_{0}^{2} \quad \text { for all } u \in H^{1}(\Omega) \text { and some } \varepsilon>0 .
\end{aligned}
$$

If the boundary of $\Omega$ and the coefficients of $a(\cdot, \cdot)$ are sufficiently smooth, the following estimates hold for all $u \in H^{3}(\Omega)$ :

$$
\begin{array}{ll}
\|u\|_{s} \leqq C\left[\|L u\|_{s-2}+|u|_{s-1 / 2}\right] & (2 \leqq s \leqq m) \\
\|u\|_{s} \leqq C\left[\left\|L^{*} u\right\|_{s-2}+|u|_{s-1 / 2}\right] & (2 \leqq s \leqq m) \\
\|u\|_{s} \leqq C\left[\|u\|_{o}+\|L u\|_{s-2}+|B u|_{s-3 / 2}\right] & (2 \leqq s \leqq m) \\
\|u\|_{s} \leqq C\left[\|u\|_{0}+\left\|L^{*} u\right\|_{s-2}+|C u|_{s-3 / 2}\right] & (2 \leqq s \leqq m) \\
|B u|_{s-3 / 2} \leqq C\|u\|_{s}, \quad|C u|_{s-3 / 2} \leqq C\|u\|_{s} & (2 \leqq s \leqq m) \\
|u|_{s-1 / 2} \leqq C\|u\|_{s} & (1 \leqq s \leqq m)
\end{array}
$$

For $L, L^{*}, B$, and $C$ compare (3.2). In the following we only require (3.4), (3.6), (3.7). Symmetry and positive definiteness of $a(\cdot, \cdot)$ is not necessary. Furthermore we need no inverse assumption and no special boundary conditions for $S_{h}$. (3.7a) implies that zero is no eigenvalue of $L u=\lambda u,\left.u\right|_{\Gamma}=0$. A similar condition for $l u=\lambda u, B u=0(\Gamma)$ (or $u+h B u=0$ ) is not required.

The following lemmata ensure the solvability of the problem $\left(3.1 \mathrm{a} / 1 \mathrm{~b}^{\prime}\right)$ and of its discretization. Moreover, Lemma 3.1 shows that the solution $u$ can be estimated independently of the penalty parameter $h$. Also, the constant $C$ of the error estimates in Lemma 3.2 does not depend on $h$. The lemmata are proved in Section 4.

Lemma 3.1: For sufficiently small $h$ the boundary value problem (3.1a), (3.1 b') has a unique solution $u=u(h)$ satisfying the estimate

$$
\|u\|_{s} \leqq C\left[\|f\|_{s-2}+|g|_{s-1 / 2}\right] \quad(2-m \leqq s \leqq m)
$$

with $C$ independent of $h, s, f, g$.

Lem ma 3.2: Let $h \in\left(0, h_{0}\right]$ with $h_{0}$ small enough. Then a unique solution $u_{h} \in S_{h}$ of

$$
A_{h}\left(u_{h}, v_{h}\right)=A_{h}\left(u, v_{h}\right) \quad \text { for all } v_{h} \in S_{h}
$$

exists and satisfies (3.10) if $u \in H^{t}(\Omega)$ :

$$
\begin{aligned}
& \left\|u_{h}-u\right\|_{s} \leqq C h^{t-8}\|u\|_{t} \quad \text { for all } 2-m \leqq s \leqq 1 \leqq t \leqq m, \\
& \left|u_{h}-u\right|_{s-1 / 2} \leqq C h^{t-8}\|u\|_{t} \quad \text { for all } 1-m \leqq s \leqq 1 \leqq t \leqq m .
\end{aligned}
$$


By virtue of Lemma 3.2 the penalty correction method $(3.5 a, b)$ is well-defined, provided $h \leqq h_{0}$. The following theorem shows that the optimal order of approximation $(=2 m-2$, cf. $(3.10))$ is obtained by $u_{h}{ }^{2 m-2}$.

Théorem 3.1: Let $h \in\left(0, h_{0}\right)$ with $h_{0}>0$ small enough. The errors of $u_{h}^{i}$ can be estimated by

$\begin{array}{ll}\left\|u_{h}^{i}-u\right\|_{s} \leqq C(i) h^{\min (t-s, i)}\left[\|f\|_{t-2}+|g|_{t-1 / 2}\right] & (2-m \leqq s \leqq 1 \leqq t \leqq m), \\ \left|u_{h}^{i}-u\right|_{s-1 / 2} \leqq C(i) h^{\min (t-s, i)}\left[\|f\|_{t-2}+|g|_{t-1 / 2}\right] & (1-m \leqq s \leqq 1 \leqq t \leqq m)\end{array}$

where $u$ is the solution of $(3.1 \mathrm{a}, \mathrm{b})$.

The proof will be given in Section 3.4 by verifying the presuppositions of the general Theorem 2.1.

Corollary 3.1: Let $h \in\left(0, h_{0}\right] \cdot h^{-1} \sum_{j=1}^{i}\left(g-u_{h}{ }^{j}\right)$ approximates $B u$ :

$$
\left|B u-h^{-1} \sum_{j=1}^{i}\left(g-u_{h}^{i}\right)\right|_{s-1 / 2} \leqq C h^{\min (\ell-s-1, i+1)}\left[\|f\|_{\ell-2}+|g|_{t-1 / 2}\right]
$$

for $1-m \leqq s \leqq 1 \leqq t \leqq m$.

Finally we remark that it is possible to obtain an error estimate $\mathcal{O}\left(h^{t-8}\right)$ even for the first iterate, if the penalty term is defined by means of the scalar product of $H^{-\sigma}(\Gamma)$.

Corollary 3.2: The solution $u_{\hbar}$ of

$$
\left.a\left(u_{h}, v_{h}\right)+h^{-1-2 \sigma}<u_{h}-g, v_{h}\right\rangle_{H^{-\sigma}(\Gamma)}=\left(f, v_{h}\right) \quad \text { for all } v_{h} \in S_{h}
$$

satisfies

$$
\left\|u_{h}-u\right\|_{s} \leqq C h^{\min (t-8,1+2 \sigma)}\left[\|f\|_{t-2}+|g|_{t-1 / 2}\right] \quad(2-m \leqq s \leqq 1 \leqq t \leqq m) .
$$

The use of the scalar product $\langle\cdot, \cdot\rangle_{H^{-\sigma}}(r)$ ist not convenient but possible. For integers $\sigma=k$ the scalar product can be defined by $\langle u, v\rangle_{H-k_{(}(\Gamma)}=\left\langle\Delta_{\Gamma}{ }^{-k} u, v\right\rangle$, where $\Delta_{\Gamma}$ is the Laplacean operator on $\Gamma$.

\subsection{Numerical solution by the multi-grid algorithm}

Let $u_{h} \in S_{h}$ have the coefficients $U_{h}$ with respect to a suitable basis: $u_{h}=P_{h} U_{h}$, where $P_{h}$ maps the coefficient vector onto $S_{h} \subset H^{1}(\Omega)$. The equations $(3.5 \mathrm{a}, \mathrm{b})$ are of the form

$$
L_{h} U_{h}=Y_{h},
$$

where the stiffness matrix $L_{h}$ is defined by

$$
\left(L_{h} U_{h}, V_{h}\right)_{h}=A_{h}\left(P_{h} U_{h}, P_{h} V_{h}\right)=a\left(P_{h} U_{h}, P_{h} V_{h}\right)+h^{-1}\left\langle P_{h} U_{h}, P_{h} V_{h}\right\rangle
$$

Here $(\cdot, \cdot)_{h}$ denotes a suitable scalar product on the coefficient vector space.

The multi-grid algorithm described in [3] is a fast iteration solving (3.13). The rate $\varrho_{h}$ of convergence is bounded by a constant $\varrho_{0}$, independent of the discretization parameter $h: \varrho_{h} \leqq \varrho_{0}$. Usually, $\varrho_{0}$ is much smaller than 1. For numerical examples compare [4]. 
As proved in [3] the convergence follows mainly from two inequalities (Eq. (3.1), (3.2) in [3]) involving norms $\|\cdot\|_{1, h},\|\cdot\|_{2, h}$ (denoted by $\|\cdot\|_{1},\|\cdot\|_{2}$ in [3]). These estimates can be shown for the choice $\left\|U_{h}\right\|_{1, h}:=\left\|P_{h} U_{h}\right\|_{0}$ and $\left\|U_{h}\right\|_{2, h}:=\left\|P_{h} U_{h}\right\|_{1}$ $+h^{-1 / 2}\left|P_{h} U_{h}\right|_{0}$

\subsection{Proof of Theorem 3.1}

In order to apply Theorem 2.1 we have to introduce the spaces $X^{a}, Y^{\alpha}, X_{h}{ }^{\alpha}, Y_{h}{ }^{a}$ and the related mappings. We set

$$
X^{\natural}=H^{8}(\Omega), Y^{s}=H^{s-2}(\Omega) \times H^{8-1 / 2}(\Gamma) .
$$

The components of $y \in Y^{s}$ are always denoted by $y^{\Omega}$ and $y^{\Gamma}: y=\left(y^{\Omega}, y^{r}\right)$. The norm of $Y^{s}$ is $\|y\|_{y^{t}}=\left\|y^{\Omega}\right\|_{s-2}+\left|y^{\Gamma}\right|_{s-1 / 2}$. The Dirichlet problem $(3.1 \mathrm{a}, \mathrm{b})$ becomes $F u$ $=y:=(f, g)$ if we define

$$
F u=\left(L u,\left.u\right|_{\Gamma}\right)
$$

for $u \in X^{s}=H^{s}(\Omega) ; s \geqq 2$.

$\bar{Y}_{\mathrm{A}}{ }^{\mathrm{i}}$ is the space of $y \in Y^{1}$ endowed with the norm

$$
\|y\|_{Y_{\mathrm{A}^{*}}}= \begin{cases}\inf \left\{h^{1-8}\left\|y_{1}\right\|_{Y^{1}}+\left\|y_{2}\right\|_{Y^{*}}: y_{1}+y_{2}=y\right\} & \text { for } s>1 \\ \|y\|_{Y^{*}}^{\prime}+h^{1-s}\|y\|_{Y^{1}} & \text { for } s \leqq 1\end{cases}
$$

Each $y \in Y_{h}^{8}$ gives rise to a solution $u_{h} \in S_{h}$ of

$$
A\left(u_{h}, v_{h}\right)=\left(y^{\Omega}, v_{h}\right)+h^{-1}\left\langle y^{\Gamma}, v_{h}\right\rangle \quad \text { for all } v_{h} \in S_{h} \text {. }
$$

Since different $y$ 's can yield the same $u_{h}$, the space $X_{h}^{s}$ does not consist of $u_{h} \in S_{h}$ but of $x=\left(u_{h}, y\right)=\left(u_{h}, y^{\Omega}, y^{r}\right)$ :

with

$$
X_{h}{ }^{8}=\left\{x=\left(u_{h}, y\right) \in S_{h} \times Y_{h}^{s} \text { satisfying (3.14) }\right\}
$$

$$
\|x\|_{x_{n}}=\|y\|_{Y_{n^{\prime}}} .
$$

$R_{h}{ }^{x} u$ is $\left(u_{h}, L u, u+h B u\right)$ with $A_{h}\left(u_{h}, v_{h}\right)=A_{h}\left(u, v_{h}\right)=\left(L u, v_{h}\right)+h^{-1}\left\langle u+h B u, v_{h}\right\rangle$ (cf. $((3.2))$ :

$$
R_{h}{ }^{x} u=F_{h}^{-1}(L u, u+h B u),
$$

where $F_{h}: X_{h}^{8} \rightarrow Y_{h}^{8}$ is defined by

$$
F_{h}\left(u_{h}, y\right)=y \text {. }
$$

By $F_{h}^{-1} y, y=(f, g)$, the solution of the finite element problem (3.5a) is described. The second mapping $F_{h}^{\prime}: X_{h}^{8} \rightarrow Y_{h}^{8}$ is

$$
F_{h}^{\prime}\left(u_{h}, y\right)=\left(y^{\circ}, u_{h} \mid r\right)
$$

according to the definition of $F$. Note that $F_{h}^{\prime}$ is not invertible, since the range of $F_{b}^{\prime}$ is a proper subspace of $Y_{h}^{8}$. Finally we set

$$
R_{h}{ }^{Y}=R_{h}{ }^{\prime}=\text { identity . }
$$

Now we can formulate that the penalty correction method is a special example of the iterated defect correction method:

Note 3.1: The penalty correction method $(3.5 \mathrm{a}, \mathrm{b})$ and the iterated defect correction. (2.4) are equivalent in the following sense: If $x_{h}{ }^{i}=\left(u_{h}{ }^{i}, y_{h}{ }^{i}\right)$ is the result of (2.4), then the sequence $u_{h}{ }^{i}$ satisfies $(3.5 \mathrm{a}, \mathrm{b})$. 
Proof: $y_{h}=R_{h}{ }^{P}(f, g)=(f, g)$ yields $x_{h}{ }^{1}=\left(u_{h}{ }^{1}, f, g\right)$ satisfying (3.14) with $y=(f, g)$. Since $F_{h}\left(x_{h}^{i+1}-x_{h}{ }^{i}\right)=R_{h}{ }^{Y}(f, g)-F_{h}{ }^{\prime} x_{h}{ }^{i}=(f, g)-\left(y_{h}{ }^{i \Omega}, u_{h}{ }^{i}\right)$ and $y_{h}{ }^{i 0}=f$ (to be proved by induction), $u_{h}^{i+1}-u_{h}^{i}$ fulfils (3.14) with $y=\left(0, g-u_{h}^{i}\right)$, hence (3.5 b) is

By the following four notes we verify the four presuppositions (2.5), (2.6a), (2.6b) (2.7) of Theorem 2.1. The definition of the norm of $\bar{X}_{h}{ }^{8}$ implies

Note 3.2: (2.5) is valid with $\sigma=0$ for all $\alpha \in[2-m, m]$.

Note 3.3: (2.6a) holds for $\beta=\varkappa=1$ and all $\alpha \in[1, m-1]$.

Proof: Let $v \in X^{a+\beta}$. Since $y=\left({R_{h}}^{Y} F-F_{h} R_{h}{ }^{X}\right) v=(L v, v)-(L v, v+h B v)$ $=(0,-h B v),(3.7 \mathrm{c})$ shows $\|y\|_{Y_{\mathrm{A}}^{\alpha}} \leqq h \mid B v\left\|_{\mathrm{s}-1 / 2} \leqq C h\right\| v\left\|_{\alpha+1}=C h\right\| v \|_{X^{\alpha+\beta}}$

Note 3.4: $(2.6 \mathrm{~b})$ is valid with $x^{\prime}=2 m-2$ for all $\alpha \in[2-m, m], \beta \geqq 0$, $\alpha+\beta \in[1, \bar{m}]$.

Proof: With $v_{h}=R_{h}{ }^{x} v$ we have $y=\left(R_{h}{ }^{P} F-F_{h}{ }^{\prime} R_{h}{ }^{X}\right) v=\left(0, v-v_{h}\right)$. Lemma $3: 2$ implies $\left|y^{\Gamma}\right|_{\alpha-1 / 2} \leqq C h^{\beta}\|v\|_{\alpha+\beta}$ and $h^{1-\alpha}\left|y^{\Gamma}\right|_{1 / 2} \leqq C h^{\beta}\|v\|_{\alpha+\beta}$ if $\alpha \leqq 1$. Thus, (2.6b) follows

Note 3.5: (2.7) holds for $\beta=x=1$ and all integers $\alpha \in[2-m, m-1]$.

Proof: (i) Consider the case of $\alpha \in[1, m-1], t=\alpha+\beta=\alpha+1 \leqq m$. Let $x_{h}=\left(w_{h}, \varphi\right) \in X_{h}{ }^{t}$ with $\omega:=\left\|x_{h}\right\|_{X_{h}{ }^{\prime}}$. We have $y=\left(F_{h}^{\prime}-F_{h}\right) x_{h}=\left(0, w_{h}-\varphi_{h}{ }^{\prime}\right)$. By definition there is $\varphi_{1}+\varphi_{2}=\varphi$ with $\left|\varphi_{1}{ }^{\Gamma}\right|_{1 / 2} \leqq 2 \omega h^{t-1},\left|\varphi_{2}\right|_{t-1 / 2} \leqq 2 \omega$. Define $w_{h, i}=F_{h}^{-1} \varphi_{i}(i=1,2)$ and let $W_{i}$ be the solutions of $L W_{i}=0, W_{i}+h B W_{i}=\varphi_{i}^{I}$ $(i=1,2)$. Lemmata 3.1 and 3.2 yield

$$
\begin{aligned}
& \left|W_{2}-\varphi_{2}{ }^{\Gamma}\right|_{t-3 / 2}=h\left|B W_{2}\right|_{t-3 / 2} \leqq C h\left\|W_{2}\right\|_{t} \leqq C^{\prime} h\left|\varphi_{2}{ }^{r}\right|_{t-1 / 2} \leqq C^{\prime \prime} \omega h \\
& h^{1-\alpha}\left[\left|w_{h, 2}-W_{2}\right|_{1 / 2}+\left|w_{h, 1}\right|_{1 / 2}+\left|\varphi_{1}\right|_{1 / 2}\right] \\
& \leqq C\left[h \|\left. W_{2}\right|_{\ell}+h^{1-\alpha}\left(\left|W_{1}\right|_{1 / 2}+\left|w_{h, 1}-W_{1}\right|_{1 / 2}\right)+\omega h\right] \leqq C^{\prime} \omega h .
\end{aligned}
$$

Splitting $y$ into $y_{1}=\left(0, w_{h, 1}-\varphi_{1}{ }^{r}+w_{h, 2}-W_{2}\right)$ and $y_{2}=\left(0, W_{2}-\varphi_{2}{ }^{r}\right)$, obtain (2.7): $\|y\|_{Y_{\mathrm{A}} \mathrm{a}^{\mathrm{a}}} \leqq C h \omega=C h\left\|x_{\mathrm{h}}\right\|_{X_{\mathrm{A}}}$ with $t=\alpha+1$.

(ii) Assume $\alpha \in[2-m, 0], t=\alpha+1 \leqq 1 . x_{h}=\left(w_{h}, \varphi\right) \in X_{h}^{t}$ satisfies $\left|\varphi^{\Gamma}\right|_{t-1 / 2}$ $+h^{1-t}\left|\varphi^{\Gamma}\right|_{I / 2} \leqq 2 \omega, \omega=\left\|x_{h}\right\|_{X_{h} \text {. }}$ The last inequality implies $\left|\varphi^{\Gamma}\right|_{s+1 / 2} \leqq C \omega h^{i-1-s \cdot}$ for $s \in[t-1,0]$. Define $W$ by $L W=0, W+h B W=\varphi^{\Gamma}(\Gamma)$. The estimates

$$
\begin{aligned}
& h^{s-\alpha}\left|w_{h}-W\right|_{s-1 / 2} \leqq C h^{1-\alpha}\left|\varphi^{\Gamma}\right|_{1 / 2} \leqq C^{\prime} h \omega \quad(\alpha \leqq s \leqq 1) \text {, } \\
& \left|W-\varphi^{\Gamma}\right|_{a-1 / 2} \leqq C h|B W|_{a-1 / 2} \leqq C^{\prime} h|W|_{a+1 / 2} \leqq C^{\prime \prime} h\left|\varphi^{r}\right|_{a+1 / 2} \leqq C^{*} h \omega, \therefore \\
& h^{1-\alpha}\left|W-\varphi^{\Gamma}\right|_{1 / 2} \leqq h^{1-a}\left(|W|_{1 / 2}+\left|\varphi^{\Gamma}\right|_{1 / 2}\right) \leqq C h \omega
\end{aligned}
$$

(cf. Lemmata $3.1,3.2,4.1)$ prove $\|y\|_{Y_{\mathrm{A}}}=\left\|\left(0, w_{h}-\varphi\right)\right\|\left\|_{Y_{\mathrm{A}}} \leqq C h \omega=C h\right\| x_{h} \|_{x_{\mathrm{A}}}$ for $t=\alpha+1 \leqq 1$, too

The following lemma connects the norms of $H^{8}(\Omega), H^{8-1 / 2}(\Gamma)$ and $X_{h}^{8}$. Its próof is given in Section 4.

Lemma 3.3: For $x_{h}=\left(u_{h}, y\right) \in X_{h}^{8}$ we have

$$
\begin{aligned}
& \left\|u_{h}\right\|_{s} \leqq C\left\|\left(u_{h}, y\right)\right\|_{x_{\mathrm{A}^{\prime}}} \quad \text { for } 2-m \leqq s \leqq 1, \\
& \left|u_{h}\right|_{8-1 / 2} \leqq C\left\|\left(u_{h}, y\right)\right\|_{x_{A^{\prime}}} \quad \text { for } 1-m \leqq s \leqq 1 .
\end{aligned}
$$

Proof of Theorem 3.1: Let $u_{h}$ the first component of $R_{h}{ }^{x} u=\left(u_{h}, L u, u+h B u\right)$. By the Notes 3.2-3.5 the estimate (2.8) of Theorem 2.1 holds. Together with Lemma 
3.3 it shows $\left\|u_{h}^{i}-u_{h}\right\|_{s} \leqq C(i) h^{i}\|u\|_{l}$ for $2-m \leqq s \leqq 1 \leqq t=s+i \leqq m$ and the same estimate for $\left|u_{h}^{i}-u_{h}\right|_{s-1 / 2}$ also for $s=1-m$. The inequality (3.7a) yields the final result

Proof of Corollary 3.1: Summing up the equations of (3.5a, b) one obtains

for all $v_{h} \in S_{h}$. Since

$$
A_{h}\left(u_{h}{ }^{i+1}, v_{h}\right)=\left(f, v_{h}\right)+h^{-1}\left\langle g+\sum_{j=1}^{i}\left(g-u_{h}{ }^{\dagger}\right), v_{h}\right)
$$

$$
A_{h}\left(u_{h}, v_{h}\right)=A_{h}\left(u, v_{h}\right)=\left(f, v_{h}\right)+h^{-1}\left\langle g+h B u, v_{h}\right\rangle
$$

for $x_{h}=\left(u_{h}, L u, u+h B u\right)=R_{h} x_{u}$ one obtains

$$
A_{h}\left(u_{h}^{i+1}-u_{h}, v_{h}\right)=h^{-1}\left\langle\varphi^{\Gamma}, v_{h}\right\rangle \text { with } \varphi^{\Gamma}=\sum_{j=1}^{i}\left(g-u_{h}^{j}\right)-h B u .
$$

In the foregoing part we estimated $x_{h}{ }^{i+1}-x_{h}=\left(u_{h}^{i+1}-u_{h}, 0, \varphi^{\Gamma}\right)$. (3.12) follows from $\left|\varphi^{\Gamma}\right|_{8-1 / 2} \leqq\left\|x_{h}^{i+1}-x_{h}\right\|_{X_{A}}$ for $s \in[1-m, 1]$

\section{Proofs of the lemmata}

\subsection{Auxiliary lemmata}

Lemma 4. 1: If Lu $=0$ then $|B u|_{s-3 / 2} \leqq C|u|_{s-1 / 2}$ for all $s \in[2-m, m]$.

Proof: The inequality holds for $s=m(\geqq 2)$ because of $(3.7 \mathrm{c}),(3.7 \mathrm{a})$. Assume $s=2-m$ and let $v \in H^{m-1 / 2}(\Gamma)$. By $\left(3.7 \mathrm{a}^{*}\right)$ there is $V \in H^{m}(\Omega)$ with $L^{*} V=0$, $\mathrm{V}=v$ on $\Gamma$. (3.2) gives

$$
\langle B u, v\rangle=a(u, V)=\langle u, C V\rangle \leqq|u|_{s-1 / 2}|C V|_{1 / 2-s} \leqq|u|_{s-1 / 2}|v|_{3 / 2-s}
$$

proving the estimate for $s=2-m$. By interpolation Lemma 4.1 follows

Lemma 4.2: If Lu $=0$ then $\|u\|_{s} \leqq C|u|_{s-1 / 2}$ for all $s \in[2-m, m]$.

Proof: (3.7a) implies the estimate for $s=m$. Let $s=2-m, v \in H^{-8}(\Omega)$ and define $w \in H^{2-s}(\Omega)$ by $L^{*} w=v,\left.w\right|_{\Gamma}=0$. (3.2) and $\left(3.7 \mathrm{a}^{*}, \mathrm{c}\right)$ imply

$$
(u, v)=a(u, w)-\langle u, C w\rangle=-\langle u, C w\rangle \leqq|u|_{s-1 / 2}|C w|_{1 / 2-s} \leqq C|u|_{s-1 / 2}\|v\|_{-s \cdot}
$$

Thus, interpolation yields Lemma 4.2

Lemma 4.3: There is $h_{0}>0$ so that

$$
\|u\|_{1}^{2} \leqq C A_{h}(u, u) \quad \text { for all } h \in\left(0, h_{0}\right), u \in H^{1}(\Omega) \text { with } L u=0 .
$$

If (3.6) holds with $C \leqq 0$, i.e., if $a(\cdot, \cdot)$ is positive, definite, then $h_{0}=\infty$ may be chosen.

Proof: (3.6b) implies

$$
\begin{aligned}
A_{h}(u, u) & =a(u, u)+h^{-1}\langle u, u\rangle \geqq \varepsilon\|u\|_{1}^{2}-C\|u\|_{0}^{2}+h^{-1}|u|_{0}^{2} \\
& \geqq \varepsilon^{\prime}\left[\|u\|_{1}^{2}+h^{-1}|u|_{0}^{2}\right]+\left(1-\varepsilon^{\prime}\right\rangle h^{-1}|u|_{0}^{8}-C\|u\|_{0}^{2},
\end{aligned}
$$

where $\varepsilon^{\prime}=\min (\varepsilon, 1 / 2)>0$. Since $\|u\|_{0}^{2} \leqq C^{\prime \prime}\|u\|_{1 / 2}^{2} \leqq C^{\prime}|u|_{0}^{2}$ by Lemma 4.2, the assertion follows if $\left(1-\varepsilon^{\prime}\right) h^{-1}-C C^{\prime} \geqq 0$, i.e., if $h \leqq h_{0}:=\left(1-\varepsilon^{\prime}\right) /\left|C C^{\prime}\right|$ or $C \leqq 0$ 
Lem ma 4.4: Choose $h_{0}$ as in Lemma 4.3 and define $\mathscr{B}$ by

$\mathscr{B} u=h_{0}^{-1} u+B u$.

For all $u \in H^{8}(\Omega)$ with $L u=0$ the following estimate holds:

$\frac{1}{C}|u|_{8-1 / 2} \leqq|\mathscr{B} u|_{s-3 / 2} \leqq C|u|_{8-1 / 2}$ for all $s \in[2-m, m], u \in H^{s}(\Omega), L u=0$.

Proof: (i) Since $h_{0}$ is fixed, the second inequality follows from Lemma 4.1. (ii) Consider the case $s=1$. Apply (3.7d), (4.1), and (3.2):

$$
|u|_{1 / 2}^{2} \leqq C^{\prime}\|u\|_{1}^{2} \leqq C A_{h_{0}}(u, u)=C\langle\mathscr{B} u, u\rangle \leqq C|\mathscr{B} u|_{-1 / 2}|u|_{1 / 2}
$$

(iii) Let $s \in[1, m]$. Using (3.7 d), (3.7 b), and Lemma 4.2 we obtain

$$
|u|_{s-1 / 2} \leqq C\|u\|_{s} \leqq C^{\prime}\left[\|u\|_{0}+|B u|_{s-3 / 2}\right] \leqq C^{\prime \prime}\left[|u|_{s-3 / 2}+|\mathscr{B} u|_{s-3,2}\right] .
$$

Hence, (ii) implies (4.2) for $1 \leqq 8 \leqq 2$. This result shows (4.2) for $2 \leqq s . \leqq 3$, etc. (iv) Let $s \in[2-m, 1], u \in H^{s-1 / 2}(\Gamma), v \in H^{1 / 2-s}(\Gamma)$. Define $V \in H^{2-8}(\Omega)$ by $L^{*} V=0$, $\mathscr{C} V:=C V+h_{0}^{-1} V=v$ on $\Gamma$. Part (iii) (with $L^{*}, C$ instead of $L, B$ ) shows $|V|_{3 / 2-8}$ $\leqq C|\mathscr{C} V|_{1 / 2-s}=C|v|_{1 / 2-s .}$. Hence,

$$
\langle u, v\rangle=A_{h_{0}}(u, V)=\langle\mathscr{B} u, V\rangle \leqq|B u|_{s-3 / 2}|V|_{3 / 2-s} \leqq C|\mathscr{B} u|_{s-3 / 2}|v|_{1 / 2-8}
$$

implies (4.2)

Lemma 4.5: Let $g \in H^{s-1 / 2}(\Gamma)$ and $h \in\left(0, h_{0} / 2\right]$. Then the boundary value problem $L u=0, u+h B u=g$ has a solution satisfying

$$
\begin{array}{lc}
\|u\|_{s} \leqq C|g|_{s-1 / 2}, & \|u\|_{s} \leqq C h^{-1}|g|_{s-3 / 2} \quad(2-m \leqq s \leqq m), \\
|u|_{s-1 / 2} \leqq C|g|_{s-1 / 2} & (1-m \leqq s \leqq m) .
\end{array}
$$

Proof: (i) The existence follows from (4.1).

(ii) Proof of (4.3 b). By virtue of (4.2) $\mathscr{B}$ can be viewed as a bounded operator from $H^{s-1 / 2}(\Gamma)$ onto $H^{s-3 / 2}(\Gamma)$. Let $s=m$ be an integer. Note that $\alpha(h) u+h \mathscr{B} u=u$ $+h B u=g$ for $\alpha(h):=1-h / h_{0} \geqq 1 / 2$. Lemma 4.4 and (4.1) show

$$
\begin{aligned}
& |u|_{m-1 / 2}^{2} \leqq C^{\prime \prime}\left|\mathscr{B}^{m-1} u\right|_{1 / 2}^{2} \leqq C^{\prime}\left\|\mathscr{B}^{m-1} u\right\|_{1}^{2} \leqq C A_{h_{0}}\left(\mathscr{B}^{m-1} u, \mathscr{B}^{m-1} u\right) \\
& \left.=C<\mathscr{B}^{m} u, \mathscr{B}^{m-1} u\right\rangle \\
& \leqq C\left\langle\mathscr{B}^{m} u, \mathscr{B}^{m-1} u+\alpha(h)^{-1} h \mathscr{B}^{m} u\right\rangle^{\prime}=C \alpha(h)^{-1}\left\langle\mathscr{B}^{m} u, \mathscr{B}^{m-1} g\right\rangle \\
& \leqq 2 C\left|\mathscr{B}^{m} u\right|_{-1 / 2}\left|\mathscr{B}^{m-1} g\right|_{1 / 2} \leqq C^{*}|u|_{m-1 / 2}|g|_{m-1 / 2},
\end{aligned}
$$

where we identify $\mathscr{B} v \in H^{t}(\Gamma)$ with the solution $V$ of $L V=0, V=\mathscr{B} v$ on $\Gamma$. Let $v \in H^{m-1 / 2}(\Gamma)$ and define $V$ by $L^{*} V=0, V+h C V=v$ on $\Gamma$. The just proved estimate (with $L^{*}, C$ instead of $L, B$ ) yields

$$
\begin{aligned}
\langle u, v\rangle & =\langle u, V+h C V\rangle=h A_{h}(u, V) \\
& =\langle g, V\rangle \leqq|g|_{1 / 2-n}|V|_{m-1 / 2} \leqq C|g|_{1 / 2-m}|v|_{m-1 / 2},
\end{aligned}
$$

hence $(4.3 \mathrm{~b})$ for $s=1-m$. Interpolation proves $(4.3 \mathrm{~b})$ for all $s$.

(iii) (4.3b) and Lemma 4.2 imply the first inequality of (4.3a).

(iv) The remaining part of $(4.3 \mathrm{a})$ follows from Lemma $4.2,(4.2)$ and $(4.3 \mathrm{~b})$ :

$$
\|u\|_{\delta} \leqq C^{\prime}|\mathscr{B} u|_{\delta-3 / 2}=C^{\prime} h^{-1}|g-\alpha(h) u|_{\delta-3 / 2} \leqq C h^{-1}|g|_{g-3 / 2}
$$


Lemma 4.6: Let $s \in[2-m, m], f \in H^{s-2}(\Omega)$ and define $u$ by $L u=f, u+h B u$ $=0$. Then $u$ satisfies

$$
\|u\|_{s}+|B u|_{s-3 / 2}+|u|_{s-1 / 2}+h^{-1}|u|_{s-3 / 2} \leqq C\|f\|_{s-2} \quad(2-m \leqq s \leqq m) .
$$

Proof: The inequalities are obvious for $s=m$. Let $s=2-m$ and $v \in B^{3 / 2-8}(\Gamma)$. Define $V$ by $L^{*} V=0, V+h C V=v$. (3.2) yields $\langle u, v\rangle=h A_{h}(u, V)=h(f, V)$. Hence, the estimates $|\langle u, v\rangle| \leqq h\|f\|_{-m}\|V\|_{m} \leqq C h|| f \|_{-m}|v|_{m-1 / 2}$ and $|\langle u, v\rangle|$ $\leqq\|f\|_{-m}|v|_{m-3 / 2}$ prove $|u|_{s-1 / 2}+h^{-1}|u|_{s-3 / 2} \leqq C\|f\|_{-m}$. The estimate of $B u$ follows from $B u=h^{-1} u$, while $\|u\|_{s} \leqq C\|f\|_{8-2}$ is shown by $(u, v)=(f, V)$ with $L^{*} V=v$. $V+h C V=0$ on $\Gamma$

\subsection{Proof of Lemmata 3.1-3.3}

Proof of Lemma 3.1: Combine the Lemmata 4.5 and 4.6.

Proof of Lemma 3.2: Set $e=u_{h}-u$. (3.6a) implies

$$
\left|A_{h}(e, v)\right| \leqq C\|e\|_{1}\|v\|_{1}+h^{-1}|e|_{1 / 2}|v|_{-1 / 2} \leqq C\|e\|_{1}\left[\|v\|_{1}+h^{-1}|v|_{-1 / 2}\right] .
$$

Let $L^{*} v=0, v+h C v=\Lambda^{28-1} e$ on $\Gamma$, where $\Lambda=\Lambda^{*}$ is defined by $\left|\Lambda^{s-1 / 2} w\right|_{0}=|w|_{s-1 / 2}$. Choosing a suitable $\varphi_{h} \in S_{h}$ one obtains

$$
\begin{aligned}
|e|_{s-1 / 2} & =\left\langle e, A^{2 s-1} e\right\rangle=h A_{h}(e, v)=h A_{h}\left(e ; v-\varphi_{h}\right) \\
& \leqq C h^{1-8}\|e\|_{1}\|v\|_{1-s} \leqq C^{\prime} h^{1-8}|e|_{s-1 / 2}\|e\|_{1},
\end{aligned}
$$

hence

$$
|e|_{s-1 / 2} \leqq C h^{1-8}\|e\|_{1} \quad(1-m \leqq s \leqq 1) .
$$

Similarly, the choice of $v$ by $L^{*} v=\Lambda^{2 s} e, v+h C v=0(\Gamma)$ with $\Lambda=\Lambda^{*}$ fulfilling $\left\|\Lambda^{s} w\right\|_{0}=\|w\|_{s}$ leads to

$$
\|e\|_{s} \leqq C h^{1-s}\|e\|_{1} \quad(2-m \leqq s \leqq 1) .
$$

From (3.6b) one concludes that

$$
\begin{aligned}
\|e\|_{1}^{2} & \leqq C A_{h}(e, e)+C^{\prime}\|e\|_{0}^{2}=C A_{h}\left(e, u-\varphi_{h}\right)+C^{\prime}\|e\|_{0}^{2} \\
& \leqq C^{\prime \prime}\left[\left(\|e\|_{1}+h^{-1}|e|_{-1 / 2}\right)\left\|u-\varphi_{h}\right\|_{1}+\|e\|_{0}^{2}\right] \\
& \leqq C^{*}\left[\|e\|_{1} h^{\ell-1}\|u\|_{\ell}+h^{2}\|e\|_{1}^{2}\right],
\end{aligned}
$$

whence $\|e\|_{1} \leqq C h^{t-1}\|u\|_{\ell}$, provided that $h$ is sufficiently small. By $(4.5 \mathrm{a}, \mathrm{b})$ all estimates of Lemma 3.2 are proved

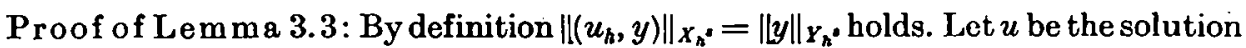
of $L u=y^{\Omega}, u+h B u=y^{\Gamma}$. Lemmata 4.5, 4.6 show $\|u\|_{B} \leqq C\|y\|_{Y_{n}}$ for $s \in[2-m, 1]$ and $|u|_{s-1 / 2} \leqq C\|y\|_{Y_{n^{*}}}$ for $s \in[1-m, 1]$. Since $\|u\|_{1} \leqq C h^{s-1}\|y\|_{Y_{A^{\prime}}}$, Lemma 3.2 implies the remaining estimates of $u-u_{h}$

\section{REFERENCES}

[1] BАвUگKa, I.: The finite element method with penalty. Nath. Comp. 27 (1973), $221-228$.

[2] Hackbosch, W.: Bemerkungen zur iterierten Defektkorrektur und zu ihrer Kombination mit Mehrgitterverfahren. Rev. Roumaine Math. Pures Appl. 26 (1981), 1319-1329. 
[3] Hack Busch, W.: On the convergence of multi-grid iterations. Beiträge zur Numer. Math. 9 (1981), 213-239.

[4] HaCkBUSOH, W.: On the multi-grid method applied to difference equations. Computing 20 (1978); $291-306$.

[5] Krwa, J. T. : New error bounds for the penalty method and extrapolation. Numer. Math. 23 (1974), 153-165.

[6] Pasciak, J.: The penalty correction method for elliptic boundary value problems. SIAM J. Numer. Anal. 16 (1979), 1046-1059.

[7] Stetrer, H. J.: The defect correction principle and discretization methods. Numer. Math. 29 (1978), 425-443.

Manuskripteingang: 08.10 .1980

\section{VERFASSER :}

Prof. Dr. Wolfoang Hackbusch

Institut fŭr Informatik und Praktische Mathematik

Christian-Albrechts-Universität Kiel

D.2300 Kiel 1, Olshausenstr. 40-60 